\title{
The Semi-Relativistic Scattering States of the Hulthén and Hyperbolic-Type Potentials
}

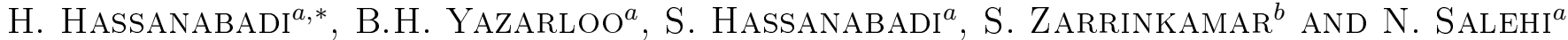 \\ ${ }^{a}$ Department of Basic Sciences, Shahrood Branch, Islamic Azad University, Shahrood, Iran \\ ${ }^{b}$ Department of Basic Sciences, Garmsar Branch, Islamic Azad University, Garmsar, Iran \\ (Received December 11, 2012; in final form March 19, 2013)
}

\begin{abstract}
In this paper, the scattering states of the spinless-Salpeter equation are investigated for Hulthén and hyperbolic-type potentials for any arbitrary l-state. Approximate analytical formulae of the wave functions and the scattering phase shifts are reported.
\end{abstract}

DOI: 10.12693 /APhysPolA.124.20

PACS: 03.65.Pm, 03.65.Nk

\section{Introduction}

The knowledge on the scattering states and related parameters such as the phase shift, transmission and reflection coefficients is a primary step in many physical studies including the nuclear and particle physics in both non-relativistic and relativistic quantum mechanics. A very challenging step in such cases is the centrifugal term which does not allow for an exact analytical solution in most cases. During the past years, various techniques have been proposed and applied to deal with such problems [1-10] and a variety of interactions, including Hulthén [11], Poschl-Teller [12] and Woods-Saxon [13] potentials have been studied. To provide a general solution for exponential potentials without having to deal with the cumbersome and rather vague numerical procedure is making use of an exponential approximation to the inverse square centrifugal term. Here, we consider the scattering states of the two-body spinless-Salpeter equation (SSE) under the Hulthén potential and an exponential-type potential which possesses interesting physical nature. These potentials have been successfully applied to nuclear, particles, atomic, condensed matter, and chemical physics [14-16].

\section{Scattering states of the arbitrary $l$-wave spinless-Salpeter equation}

The SSE for two particles interacting in a spherically symmetric potential in the center of mass system appears as [17]:

$$
\begin{aligned}
& {\left[\sum_{i=1,2}\left(\sqrt{-\Delta+m_{i}^{2}}-m_{i}\right)+V(r)-E_{n, l}\right] \chi(\boldsymbol{r})=0} \\
& \Delta=\nabla^{2}
\end{aligned}
$$

where $\chi(\boldsymbol{r})=R_{n l}(r) Y_{l m}(\theta, \varphi)$. In the case of heavy interacting particles, we can write $(\hbar=c=1)$ :

$$
\begin{aligned}
& {\left[-\frac{1}{2 \mu} \frac{\mathrm{d}^{2}}{\mathrm{~d} r^{2}}+\frac{l(l+1)}{2 \mu r^{2}}+W_{n l}(r)-\frac{W_{n l}^{2}(r)}{2 \tilde{m}}\right] \psi_{n l}(r)} \\
& \quad=0,
\end{aligned}
$$

where we have applied the well-known transformation

*corresponding author; e-mail: h.hasanabadi@shahroodut.ac.ir

$$
R_{n, l}(r)=\psi_{n, l}(r) / r
$$

and

$$
\begin{aligned}
& W_{n l}(r)=V(r)-E_{n l}, \\
& \tilde{m}=\eta^{3} / \mu^{2}=\left(m_{1} m_{2} \mu\right) /\left(m_{1} m_{2}-3 \mu^{2}\right) . \\
& \text { 2.1. The Hulthén potential }
\end{aligned}
$$

The Hulthén potential is given by

$$
V(r)=-\frac{V_{0}}{\mathrm{e}^{\alpha r}-1},
$$

where $V_{0}$ and $\alpha$ are constant coefficients. Substitution of Eq. (6) in Eq. (2) gives

$$
\begin{aligned}
& \left(\frac{\mathrm{d}^{2}}{\mathrm{~d} r^{2}}-A^{\prime} \frac{\mathrm{e}^{-2 \alpha r}}{\left(1-\mathrm{e}^{-\alpha r}\right)^{2}}+B^{\prime} \frac{\mathrm{e}^{-\alpha r}}{1-\mathrm{e}^{-\alpha r}}+C^{\prime}\right) \psi_{n, l}(r) \\
& \quad=0
\end{aligned}
$$

where we make use of the elegant approximation to get rid of the centrifugal term [18, 19]

$$
\frac{1}{r^{2}} \approx 4 \alpha^{2} \frac{\mathrm{e}^{-2 \alpha r}}{\left(1-\mathrm{e}^{-\alpha r}\right)^{2}}
$$

and

$$
\begin{aligned}
A^{\prime} & =-\frac{\mu V_{0}^{2}}{\tilde{m}}+4 l(l+1) \alpha^{2}, \\
B^{\prime} & =2 \mu V_{0}+\frac{2 \mu V_{0} E_{n, l}}{\tilde{m}}, \\
C^{\prime} & =\frac{\mu E_{n, l}^{2}}{\tilde{m}}+2 \mu E_{n, l} .
\end{aligned}
$$

By using the new variable $y=1-\exp (-\alpha r)$ we have

$$
\begin{aligned}
& \left(y(1-y) \frac{\mathrm{d}^{2}}{\mathrm{~d} y^{2}}-y \frac{\mathrm{d}}{\mathrm{d} y}-\frac{A^{\prime}}{\alpha^{2}} \frac{1-y}{y}+\frac{C^{\prime}}{\alpha^{2}} \frac{y}{1-y}+\frac{B^{\prime}}{\alpha^{2}}\right) \\
& \quad \times \psi_{n, l}(y)=0 .
\end{aligned}
$$

In order to obtain the solution to Eq. (10), we take the radial wave functions of the form

$$
\psi_{n, l}(y)=y^{\gamma}(1-y)^{\lambda} h_{n, l}(y) \text {. }
$$

Substitution of Eq. (11) into Eq. (10) allows us to obtain the following differential equation:

$$
\begin{aligned}
& {\left[y(1-y) \frac{\mathrm{d}^{2}}{\mathrm{~d} y^{2}}+\left(\eta_{1}-\left(1+\eta_{2}+\eta_{3}\right) y\right) \frac{\mathrm{d}}{\mathrm{d} y}-\eta_{2} \eta_{3}\right]} \\
& \quad \times h_{n, l}(y)=0,
\end{aligned}
$$

whose solutions are nothing but the hypergeometric functions [20]:

$$
h_{n, l}(y)={ }_{2} F_{1}\left(\eta_{2}, \eta_{3}, \eta_{1} ; y\right) \text {, }
$$


where

$$
\begin{aligned}
& \lambda^{2}=-\frac{C^{\prime}}{\alpha^{2}} \Rightarrow \lambda=-\mathrm{i} \frac{k}{\alpha}, \quad k=\sqrt{C^{\prime}} \\
& \gamma^{2}-\gamma=\frac{A^{\prime}}{\alpha^{2}} \Rightarrow \gamma=\frac{1}{2}\left(1 \pm \sqrt{1+\frac{4 A^{\prime}}{\alpha^{2}}}\right)
\end{aligned}
$$

and

$$
\begin{aligned}
\eta_{2} & =\frac{1}{2}\left(2 \gamma+2 \lambda+\sqrt{\frac{4\left(A^{\prime}+B^{\prime}-C^{\prime}\right)}{\alpha^{2}}}\right) \\
& =\frac{1}{2}\left(2 \gamma-\frac{2 i k}{\alpha}+\sqrt{\frac{4\left(A^{\prime}+B^{\prime}-C^{\prime}\right)}{\alpha^{2}}}\right), \\
\eta_{3} & =\frac{1}{2}\left(2 \gamma+2 \lambda-\sqrt{\frac{4\left(A^{\prime}+B^{\prime}-C^{\prime}\right)}{\alpha^{2}}}\right) \\
& =\frac{1}{2}\left(2 \gamma-\frac{2 i k}{\alpha}-\sqrt{\frac{4\left(A^{\prime}+B^{\prime}-C^{\prime}\right)}{\alpha^{2}}}\right),
\end{aligned}
$$$$
\eta_{1}=2 \gamma
$$

Using relation (11), we may write down the radial wave functions as

$$
\psi_{n, l}(y)=N_{n, l} y^{\gamma}(1-y)_{2}^{\lambda} F_{1}\left(\eta_{2}, \eta_{3}, \eta_{1} ; y\right)
$$

or

$$
\psi_{n, l}(r)=N_{n, l} \mathrm{e}^{\mathrm{i} k r}\left(1-\mathrm{e}^{-\alpha r}\right)_{2}^{\gamma} F_{1}\left(\eta_{2}, \eta_{3}, \eta_{1} ; 1-\mathrm{e}^{-\alpha r}\right) .
$$

We write the asymptotic form of the wave function given in Eq. (17) to obtain the scattering phase shifts and the normalized factor. For this purpose, we use the property of the hypergeometric functions [12]

$$
\begin{aligned}
& { }_{2} F_{1}(a, b ; c ; 0)=1, \\
& { }_{2} F_{1}(a, b ; c ; s)=\frac{\Gamma(c) \Gamma(c-a-b)}{\Gamma(c-a) \Gamma(c-b)} \\
& \quad \times{ }_{2} F_{1}(a, b ; a+b-c+1 ; 1-s) \\
& \quad+(1-s)^{c-a-b} \frac{\Gamma(c) \Gamma(a+b-c)}{\Gamma(a) \Gamma(b)} \\
& \quad \times{ }_{2} F_{1}(c-a, c-b ; c-a-b+1 ; 1-s) .
\end{aligned}
$$

By using Eq. (18), for $r \rightarrow \infty$ we can write

${ }_{2} F_{1}\left(\eta_{2}, \eta_{3}, \eta_{1} ; 1-\mathrm{e}^{-\alpha r}\right)$

$$
\begin{aligned}
& =\Gamma\left(\eta_{1}\right)\left(\frac{\Gamma\left(\eta_{1}-\eta_{2}-\eta_{3}\right)}{\Gamma\left(\eta_{1}-\eta_{2}\right) \Gamma\left(\eta_{1}-\eta_{3}\right)}\right. \\
& \left.+\left(\frac{\Gamma\left(\eta_{1}-\eta_{2}-\eta_{3}\right)}{\Gamma\left(\eta_{1}-\eta_{2}\right) \Gamma\left(\eta_{1}-\eta_{3}\right)}\right)^{*} \mathrm{e}^{-2 \mathrm{i} \alpha k r}\right)
\end{aligned}
$$

where

$$
\begin{aligned}
& \eta_{1}-\eta_{2}-\eta_{3}=2 \mathrm{i} k / \alpha=\left(\eta_{2}+\eta_{3}-\eta_{1}\right)^{*} \\
& \eta_{1}-\eta_{2}=\eta_{3}^{*}, \eta_{1}-\eta_{3}=\eta_{2}^{*}
\end{aligned}
$$

Taking

$$
\begin{aligned}
& \frac{\Gamma\left(\eta_{1}-\eta_{2}-\eta_{3}\right)}{\Gamma\left(\eta_{1}-\eta_{2}\right) \Gamma\left(\eta_{1}-\eta_{3}\right)} \\
& \quad=\left|\frac{\Gamma\left(\eta_{1}-\eta_{2}-\eta_{3}\right)}{\Gamma\left(\eta_{1}-\eta_{2}\right) \Gamma\left(\eta_{1}-\eta_{3}\right)}\right| \mathrm{e}^{\mathrm{i} \delta^{\prime}}
\end{aligned}
$$

simplified Eq. (19) as

$$
\begin{aligned}
& { }_{2} F_{1}\left(\eta_{2}, \eta_{3}, \eta_{1} ; 1-\mathrm{e}^{-\alpha r}\right) \\
& =\Gamma\left(\eta_{1}\right)\left|\frac{\Gamma\left(\eta_{1}-\eta_{2}-\eta_{3}\right)}{\Gamma\left(\eta_{1}-\eta_{2}\right) \Gamma\left(\eta_{1}-\eta_{3}\right)}\right| \\
& \quad \times \mathrm{e}^{-\mathrm{i} k r}\left(\mathrm{e}^{\mathrm{i}\left(k r+\delta^{\prime}\right)}+\mathrm{e}^{-\mathrm{i}\left(k r+\delta^{\prime}\right)}\right) .
\end{aligned}
$$

Therefore, we have the asymptotic form of the (17) for $r \rightarrow \infty$

$$
\begin{aligned}
& \psi_{n, l}(r)=2 N_{n, l} \Gamma\left(\eta_{1}\right)\left|\frac{\Gamma\left(\eta_{1}-\eta_{2}-\eta_{3}\right)}{\Gamma\left(\eta_{1}-\eta_{2}\right) \Gamma\left(\eta_{1}-\eta_{3}\right)}\right| \\
& \quad \times \sin \left(k r+\frac{\pi}{2}+\delta^{\prime}\right) .
\end{aligned}
$$

From the boundary condition of $[4,19]$ :

$$
r \rightarrow \infty \Rightarrow \psi(\infty) \rightarrow 2 \sin \left(k r-\frac{l \pi}{2}+\delta_{l}\right)
$$

and a comparison with Eq. (22), the phase shifts and the normalized constant can be found as

$$
\begin{gathered}
\delta_{l}=\frac{\pi}{2}+\frac{l \pi}{2}+\delta^{\prime}=\frac{\pi}{2}+\frac{l \pi}{2} \\
+\arg \left(\frac{\Gamma\left(\eta_{1}-\eta_{2}-\eta_{3}\right)}{\Gamma\left(\eta_{1}-\eta_{2}\right) \Gamma\left(\eta_{1}-\eta_{3}\right)}\right), \\
N_{n, l}=\frac{1}{\Gamma\left(\eta_{1}\right)}\left|\frac{\Gamma\left(\eta_{1}-\eta_{2}\right) \Gamma\left(\eta_{1}-\eta_{3}\right)}{\Gamma\left(\eta_{1}-\eta_{2}-\eta_{3}\right)}\right| . \\
\text { 2.2. The hyperbolic-type potential }
\end{gathered}
$$

Now, we study the hyperbolic type potential $[17,21]$ and [22]:

$$
V(r)=V_{0}[1-\operatorname{coth}(\alpha r)]
$$

Substitution of Eq. (25) into Eq. (2) gives

$$
\begin{aligned}
& {\left[\frac{\mathrm{d}^{2}}{\mathrm{~d} r^{2}}+D \frac{\mathrm{e}^{-2 \alpha r}}{\left(1-\mathrm{e}^{-2 \alpha r}\right)^{2}}+G \frac{1+\mathrm{e}^{-2 \alpha r}}{1-\mathrm{e}^{-2 \alpha r}}+H\right] \psi_{n, l}(r)} \\
& \quad=0
\end{aligned}
$$

where

$$
\begin{aligned}
& D=\frac{4 \mu V_{0}^{2}}{\tilde{m}}-4 l(l+1) \alpha^{2}, \\
& G=2 \mu V_{0}-\frac{2 \mu V_{0}}{\tilde{m}}\left(V_{0}-E_{n, l}\right), \\
& H=\frac{\mu\left(V_{0}-E_{n, l}\right)^{2}}{\tilde{m}}-2 \mu\left(V_{0}-E_{n, l}\right)+\frac{\mu V_{0}^{2}}{\tilde{m}} .
\end{aligned}
$$

In deriving Eq. (26), we have used the Pekeris-type approximation in Eq. (8). Applying the two transformations, i.e., $x=1-\exp (-2 \alpha r)$ and $\psi_{n, l}(x)=x^{\xi_{1}}(1-$ $x)^{\xi_{2}} f_{n, l}(x)$ we write Eq. (26) into the form of

$$
\begin{aligned}
& {\left[x(1-x) \frac{\mathrm{d}^{2}}{\mathrm{~d} x^{2}}+\left(\gamma_{3}-\left(1+\gamma_{1}+\gamma_{2}\right) x\right) \frac{\mathrm{d}}{\mathrm{d} x}-\gamma_{1} \gamma_{2}\right]} \\
& \quad \times f_{n, l}(x)=0
\end{aligned}
$$

with

$$
\begin{aligned}
& \xi_{2}^{2}=-\frac{G+H}{4 \alpha^{2}} \Rightarrow \xi_{2}= \pm \frac{\mathrm{i} k}{2 \alpha}, \quad k=\sqrt{G+H}, \\
& \xi_{1}^{2}-\xi_{1}=-\frac{D}{4 \alpha^{2}} \Rightarrow \xi_{1}=\frac{1}{2}\left(1 \pm \sqrt{1-\frac{D}{\alpha^{2}}}\right)
\end{aligned}
$$

and 


$$
\begin{aligned}
\gamma_{1} & =\frac{1}{2}\left(2 \xi_{1}+2 \xi_{2}+\sqrt{\frac{G-H}{\alpha^{2}}}\right) \\
& =\frac{1}{2}\left(2 \xi_{1}-\frac{\mathrm{i} k}{\alpha}+\sqrt{\frac{G-H}{\alpha^{2}}}\right), \\
\gamma_{2} & =\frac{1}{2}\left(2 \xi_{1}+2 \xi_{2}-\sqrt{\frac{G-H}{\alpha^{2}}}\right) \\
& =\frac{1}{2}\left(2 \xi_{1}-\frac{\mathrm{i} k}{\alpha}-\sqrt{\frac{G-H}{\alpha^{2}}}\right), \\
\gamma_{3} & =2 \xi_{1} .
\end{aligned}
$$

The solution of Eq. (28) can be expressed in hypergeometric functions as

$$
f_{n, l}(x)={ }_{2} F_{1}\left(\gamma_{1}, \gamma_{2}, \gamma_{3} ; x\right) .
$$

Therefore the wave functions of the system are

$$
\begin{gathered}
\psi_{n, l}(r)=N_{n, l} \mathrm{e}^{\mathrm{i} k r}\left(1-\mathrm{e}^{-2 \alpha r}\right)^{\xi_{1}} \\
\times_{2} F_{1}\left(\gamma_{1}, \gamma_{2}, \gamma_{3} ; 1-\mathrm{e}^{-2 \alpha r}\right) .
\end{gathered}
$$

To obtain the normalized constant and the phase shifts we once again use the properties of hypergeometric functions given in Eq. (18). Thus, ${ }_{2} F_{1}\left(\gamma_{1}, \gamma_{2}, \gamma_{3} ; 1-\mathrm{e}^{-2 \alpha r}\right)$ for $r \rightarrow \infty$ can be written as

$$
\begin{aligned}
& { }_{2} F_{1}\left(\gamma_{1}, \gamma_{2}, \gamma_{3} ; 1-\mathrm{e}^{-2 \alpha r}\right) \\
& \stackrel{(r \rightarrow \infty)}{=} \Gamma\left(\gamma_{3}\right)\left|\frac{\Gamma\left(\gamma_{3}-\gamma_{1}-\gamma_{2}\right)}{\Gamma\left(\gamma_{3}-\gamma_{1}\right) \Gamma\left(\gamma_{3}-\gamma_{2}\right)}\right| \\
& \quad \times \mathrm{e}^{-\mathrm{i} k r}\left[\mathrm{e}^{\mathrm{i}\left(k r+\delta^{\prime}\right)}+\mathrm{e}^{-\mathrm{i}\left(k r+\delta^{\prime}\right)}\right],
\end{aligned}
$$

where

$$
\begin{aligned}
& \gamma_{3}-\gamma_{1}-\gamma_{2}=\frac{\mathrm{i} k}{\alpha}=\left(\gamma_{1}+\gamma_{2}-\gamma_{3}\right)^{*}, \\
& \gamma_{3}-\gamma_{1}=\gamma_{2}^{*}, \\
& \gamma_{3}-\gamma_{2}=\gamma_{1}^{*}, \\
& \frac{\Gamma\left(\gamma_{3}-\gamma_{1}-\gamma_{2}\right)}{\Gamma\left(\gamma_{3}-\gamma_{1}\right) \Gamma\left(\gamma_{3}-\gamma_{2}\right)}=\left|\frac{\Gamma\left(\gamma_{3}-\gamma_{1}-\gamma_{2}\right)}{\Gamma\left(\gamma_{3}-\gamma_{1}\right) \Gamma\left(\gamma_{3}-\gamma_{2}\right)}\right| \mathrm{e}^{\mathrm{i} \delta^{\prime}} .
\end{aligned}
$$

Substitution of Eq. (33) into Eq. (32) leads to

$$
\begin{aligned}
& \psi_{n, l}(r)=N_{n, l} \Gamma\left(\gamma_{3}\right)\left|\frac{\Gamma\left(\gamma_{3}-\gamma_{1}-\gamma_{2}\right)}{\Gamma\left(\gamma_{3}-\gamma_{1}\right) \Gamma\left(\gamma_{3}-\gamma_{2}\right)}\right| \\
& \times\left[\mathrm{e}^{\mathrm{i}\left(k r+\delta^{\prime}\right)}+\mathrm{e}^{-\mathrm{i}\left(k r+\delta^{\prime}\right)}\right] \\
& =N_{n, l} \Gamma\left(\gamma_{3}\right)\left|\frac{\Gamma\left(\gamma_{3}-\gamma_{1}-\gamma_{2}\right)}{\Gamma\left(\gamma_{3}-\gamma_{1}\right) \Gamma\left(\gamma_{3}-\gamma_{2}\right)}\right| \\
& \quad \times 2 \sin \left(k r+\delta^{\prime}+\frac{\pi}{2}\right) .
\end{aligned}
$$

The normalization constant and the phase shift in this case respectively are

$$
\begin{aligned}
& N_{n, l}=\frac{1}{\Gamma\left(\gamma_{3}\right)}\left|\frac{\Gamma\left(\gamma_{3}-\gamma_{1}\right) \Gamma\left(\gamma_{3}-\gamma_{2}\right)}{\Gamma\left(\gamma_{3}-\gamma_{1}-\gamma_{2}\right)}\right|, \\
& \delta_{l}=\frac{\pi}{2}+\frac{l \pi}{2}+\delta^{\prime}=(l+1) \frac{\pi}{2} \\
& \quad+\arg \left(\frac{\Gamma\left(\gamma_{3}-\gamma_{1}-\gamma_{2}\right)}{\Gamma\left(\gamma_{3}-\gamma_{1}\right) \Gamma\left(\gamma_{3}-\gamma_{2}\right)}\right) .
\end{aligned}
$$

\section{Conclusion}

We have studied the scattering states of the SSE for the Hulthén and hyperbolic-type potentials. The normalized wave functions of scattering states and the formula of phase shifts are presented after some approximation to the centrifugal term. Results are useful in quantum mechanics and particle physics.

\section{Acknowledgments}

It is a great pleasure for authors to thank the kind referee for his many useful comments on the manuscript.

\section{References}

[1] G.F. Wei, S.H. Dong, Eur. Phys. J. A 46, 207 (2010).

[2] G.F. Wei, S.H. Dong, Eur. Phys. J. A 43, 185 (2010).

[3] S. Hassanabadi, A.A. Rajabi, B.H. Yazarloo, S. Zarrinkamar, H. Hassanabadi, Advances in High Energy Physics 2012, ID 804652.

[4] G.F. Wei, X.Y. Liu, W.L. Chen, Int. J. Theor. Phys. 48, 1649 (2009).

[5] G.F. Wei, C.Y. Long, S.H. Dong, Phys. Lett. A 372, 2592 (2008).

[6] H. Hassanabadi, B.H. Yazarloo, S. Zarrinkamar, A.A. Rajabi, Phys. Rev. C 84, 064003 (2011).

[7] A. Arda, R. Sever, J. Math. Phys. 52, 092101 (2011).

[8] S.H. Dong, M. Cruz-Irisson, J. Math. Chem. 50, 881 (2012).

[9] L.L. Lu, B.H. Yazarloo, S. Zarrinkamar, G. Liu, H. Hassanabadi, Few-Body Syst. 53, 573 (2012).

[10] A. Arda, O. Aydogdu, R. Sever, J. Phys. A, Math. Theor. 43, 425204 (2010).

[11] C.Y. Chen, D.S. Sun, C.L. Liu, F.L. Lu, Commun. Theor. Phys. 55, 399 (2011).

[12] C.Y. Chen, F.L. Lu, You Yuan, Chin. Phys. B 21, 030302 (2012).

[13] C. Rojas, V.M. Villalba, Phys. Rev. A 71, 052101 (2005).

[14] M. Jameel, J. Phys. A, Math. Gen. 19, 1967 (1986).

[15] R. Barnan, R. Rajkumar, J. Phys. A, Math. Gen. 20, 3051 (1987).

[16] R.L. Hall, J. Phys. A, Math. Gen. 25, 1373 (1992).

[17] H. Hassanabadi, S. Zarrinkamar, B.H. Yazarloo, Chin. J. Phys. 50, 788 (2012).

[18] W.C. Qiang, S.H. Dong, Phys. Lett. A 368, 13 (2007).

[19] S.H. Dong, W.C. Qiang, G.H. Sun, V.B. Bezerra, J. Phys. A 40, 10535 (2007).

[20] L.D. Landau, E.M. Lifshitz, Quantum Mechanics, Non-Relativistic Theory, 3rd ed., Pergamon, New York 1977.

[21] S.H. Dong, A. Gonzalez-Cisneros, Ann. Phys. 323, 1136 (2008).

[22] S. Dong, S.G. Miranda, F.M. Enriquez, S.H. Dong, Mod. Phys. Lett. B 22, 483 (2008). 